hep-ph/0402113

February, 2004

UMD-PP-04-019

\title{
CKM CP Violation in a Minimal SO(10) Model for Neutrinos and Its Implications
}

\author{
Bhaskar Dutta*, Yukihiro Mimura* and R.N. Mohapatra ${ }^{\dagger}$ \\ * Department of Physics, University of Regina, \\ Regina, Saskatchewan S4S 0A2, Canada \\ ${ }^{\dagger}$ Department of Physics, University of Maryland, \\ College Park, MD 20742, USA
}

\begin{abstract}
A minimal supersymmetric SO(10) model with one 10 and one $\mathbf{1 2 6}$ Higgs superfield has recently been shown to predict all neutrino mixings as well as the solar mass difference squared in agreement with observations. Two assumptions critical to the predictivity and success of the model are that: (i) the superpotential includes only renormalizable terms, thereby limiting the number of free parameters and (ii) the triplet term in the type II seesaw formula for neutrino mass dominates, leading to the sum rule $M_{\nu}=c\left(M_{d}-M_{e}\right)$ that is responsible for large mixings. However, CKM CP phase is constrained to be in the second or third quadrant requiring a significant non-CKM component to $\mathrm{CP}$ violation to explain observations. We revisit this issue using type I seesaw formula for neutrino masses and obtain the following results: (i) we show that the above sumrule responsible for large mixing angles can also emerge in type I seesaw models; the detailed predictions are however not compatible with present data for any choice of CP phases. (ii) We then show that addition of a nonrenormalizable term restores compatibility with neutrino data and CKM CP violation both in type I and type II cases. We further find that (iii) the MSSM parameter $\tan \beta \geq 30$ in the type I model and (iv) lepton flavor violation and lepton electric dipole moments which are accessible to proposed experiments in both type I and type II models. We also discuss the unification of the gauge couplings in type I model which requires an intermediate scale.
\end{abstract}




\section{Introduction}

The observations involving the solar and atmospheric neutrinos together with those using the accelerator and reactor neutrinos have now conclusively established that neutrinos have mass and they mix among themselves. In conjunction with the negative results from $\mathrm{CHOOZ}$ and PALO-VERDE reactor experiments, a reasonably clear outline of the mixing pattern among the three generations of neutrinos has emerged. Of the three angles needed to characterize these mixings, $\theta_{12}, \theta_{23}$ and $\theta_{13}$, the first two, responsible for solar and atmospheric neutrino deficits respectively, are large, and the third corresponding to reactor neutrinos is small [1, 2]. One of the major experimental issues in neutrino physics now is to make the knowledge of these angles more precise.

Foremost among the theoretical challenges that have already emerged from these discoveries are, first an understanding of the smallness of neutrino masses and second, understanding the vastly different pattern of mixings among neutrinos from the quarks. Specifically, a key question is whether it is possible to reconcile the large neutrino mixings with small quark mixings in grand unified frameworks that unify quarks and leptons.

The first challenge, i.e. the lightness of neutrino masses is elegantly answered by the seesaw mechanism [3] which requires an extension of the standard model that includes heavy right handed neutrinos. The light neutrino mass matrix is obtained by integrating out heavy right handed neutrinos and one gets

$$
\mathcal{M}_{\nu}^{\mathrm{I}}=-M_{\nu}^{D} M_{R}^{-1}\left(M_{\nu}^{D}\right)^{T}
$$

where $M_{\nu}^{D}$ is the Dirac neutrino mass matrix and $M_{R}$ is the right handed Majorana mass matrix. It is therefore important to explore whether one can answer the second puzzle of large neutrino mixings within the seesaw framework.

The above formula for the neutrino mass matrix is called the type I seesaw formula. The right handed Majorana mass scale, $v_{R}$, is almost determined by the mass squared difference needed to understand the atmospheric neutrino data to be around $10^{14} \mathrm{GeV}$, if we assume that the Dirac neutrino mass is same as up-type quark mass. Before proceeding to discuss the implications of this large right handed neutrino mass, let us discuss the nature of the seesaw formula.

We will consider a class of models where the right handed neutrino mass is not put in by hand but arises from a renormalizable coupling of the form $f N N \Delta_{R}$, where $N$ is a right handed 
neutrino, $f$ is a coupling constant and $\Delta_{R}$ is a Higgs field whose vacuum expectation value (vev) gives mass to the right handed neutrino. This is a natural feature of models with asymptotic parity conservation, such as those based on $S U(2)_{L} \times S U(2)_{R} \times U(1)_{B-L}$ or any higher gauge group such as $\mathrm{SO}(10)$ ), where the $\Delta_{R}$ field is part of an $S U(2)_{R}$ triplet field. Parity invariance then implies that we also have an $f \nu \nu \Delta_{L}$ coupling term as a parity partner of the $N N \Delta_{R}$ coupling. In this class of theories, whenever $\Delta_{R}$ acquires a vev, so does $\Delta_{L}$ and they are related by the formula $\left\langle\Delta_{L}\right\rangle \equiv v_{L}=\frac{v_{w}^{2}}{\lambda v_{R}}$, where $v_{w}$ is a weak scale and $v_{R}$ is the $\Delta_{R}$ vev and $\lambda$ is a coupling constant in the Higgs potential. The $\Delta_{L}$ vev contributes a separate seesaw suppressed Majorana mass to the neutrino leading to a modified seesaw formula for neutrino masses given below.

$$
\mathcal{M}_{\nu}^{\mathrm{II}}=M_{L}-M_{\nu}^{D} M_{R}^{-1}\left(M_{\nu}^{D}\right)^{T}
$$

where $M_{L}=f v_{L}$ and $M_{R}=f v_{R}$. This formula for the neutrino mass matrix is called the type II seesaw formula [4. In the case where right handed Majorana masses are heavy enough, the second term in the type II seesaw formula can be negligible, and the first term, $M_{L}=f v_{L}$, is dominant. We will call this pure type II seesaw. When both terms are comparable, we will call this mixed type II seesaw.

Coming to the large scale for right handed neutrino mass (i.e. $10^{14} \mathrm{GeV}$ or higher), we note that it suggests supersymmetric grand unified theory (GUT) as a natural framework to study neutrino masses, since experimental data suggests the gauge coupling unification scale in the minimal supersymmetric extension of the Standard Model (MSSM) to be, $M_{G} \sim 2 \times 10^{16} \mathrm{GeV}$, which is close to the seesaw scale. The minimal grand unification model for neutrinos is the one which is based on the $\mathrm{SO}(10)$ group since all standard model fermions and the right handed neutrino fit into the 16-dimensional representation of $\mathrm{SO}(10)$, resulting not only in a complete unification of the quarks and leptons but also yielding possible relations between the quark and lepton mass matrices. One may therefore hope that the neutrino oscillation parameters might be predictable in an $\mathrm{SO}(10)$ theory.

There are two simple routes to realistic $\mathrm{SO}(10)$ model building. In the first class, one may have smaller representations for the Higgs fields like 10 and 16 multiplets. In this case, the nonrenormalizable terms are added to the superpotential to implement the seesaw mechanism. These models have the disadvantage that they break R-parity which then induces rapid proton decay at an unacceptable level.

An alternative is to introduce both $\mathbf{1 0}$ and $\overline{\mathbf{1 2 6}}$ Higgs multiplets to give fermion masses. 
In this class of models, the R-parity is an automatic symmetry of the model. This naturally prevents the baryon and lepton number violating terms that give rise to rapid proton decay and also guarantees a naturally stable supersymmetric dark matter.

In $\mathrm{SO}(10)$ models of this type, both the $\Delta_{L}$ and $\Delta_{R}$ fields are part of the $\overline{\mathbf{1 2 6}}$ multiplet. The above mentioned couplings that contribute to seesaw formula arise from the couplings of 16 matter spinors to $\overline{\mathbf{1 2 6}}$ multiplet. If the rest of the Higgs sector is appropriately chosen, this leads to type II seesaw formula for neutrinos.

An interesting class of renormalizable $\mathrm{SO}(10)$ models with $\overline{\mathbf{1 2 6}}$ was proposed in Ref. [5] where it was shown that if in addition to the Higgs multiplets that break SO(10) and do not couple to fermions, one chooses only one $\mathbf{1 0}$ and one $\overline{\mathbf{1 2 6}}$ Higgs field, then the model provides a completely realistic description of the charged fermion sector of the standard model and is very predictive in the neutrino sector without any extra symmetry assumptions. The reason for this is that all the Yukawa parameters of the model are determined by the quark and charged lepton sector. This model is called the minimal renormalizable $\mathrm{SO}(10)$ model, since unlike the SO(10) models with 16 Higgs fields, it does not add any nonrenormalizable terms to the superpotential. The presence of the $\overline{\mathbf{1 2 6}}$ representation that allows the neutrino flavor structure to be related to other fermion mass matrices is at the heart of the predictivity of the model.

In the Refs. [6, 7, 8], this model was analyzed using the type I seesaw formula to see whether the neutrino oscillation parameters predicted would agree with observations. They found that the atmospheric and solar mixings can be large, and the phenomenological predictions are studied [9]. However they found that mass squared ratio for the solar and atmospheric data is $\Delta m_{\odot}^{2} / \Delta m_{A}^{2} \simeq 0.2$. This result is incompatible with the recent combined data analysis of the solar mixing angle and mass squared ratio [2].

A new approach to discussing neutrinos in this model was presented during the past year. Considering only the second and third generation sector, the authors of Ref. [10] pointed out that the type II seesaw in the minimal SO(10) model can provide a natural way to understand the maximal atmospheric mixing due to the convergence of bottom quark and tau lepton masses when extrapolated to GUT scale. The reason for this that in the the pure-type II seesaw case, the $\overline{\mathbf{1 2 6}}$ Higgs coupling leads the neutrino mass matrix to be proportional to $M_{d}-M_{e}$, where $M_{d}$ is a down-type quark mass matrix and $M_{e}$ is a charged-lepton mass matrix. It is then easy to see that $b-\tau$ mass convergence makes the 3-3 entry of the neutrino mass matrix very small, leading to large atmospheric mixing angle. While this observation was interesting, a priori, it was not clear if this would survive once the model is extended to include three generations. It 
was however shown in Ref.[11], that the same $b$ - $\tau$ mass convergence that led to large atmospheric mixing, also leads to large solar mixing while keeping the 1-3 mixing angle small as required by data. It also predicts the ratio of solar to atmospheric mass squared difference i.e. $\Delta m_{\odot}^{2} / \Delta m_{A}^{2}$ to be in the right range. This establishes that the minimal renormalizable $\mathrm{SO}(10)$ models with 126 Higgs provide a very interesting way to understand neutrino masses and mixings within a complete quark-lepton unified framework.

In Ref.[11], all the CP phases, including the Kobayashi-Maskawa (KM) phase, were set to zero (or 180 degree) and it was assumed that all CP violating effects owe their origin to the SUSY breaking sector. In Ref. [12, the pure type II model including all CP phases was analyzed, and it was found that one can maintain the predictivity of the model despite the appearance of the phases; but the KM phase must be in the second quadrant in the $\rho-\eta$ plane to maintain the neutrino predictions (where we have adopted the Wolfenstein parameterization for the CKM matrix). This implies that the model is substantially different in CP violating sector from the standard CKM model [13] where the KM phase must be in the first quadrant. Such non-CKM CP violation, for instance, could be in the squark mixings. Phenomenologically speaking, there is nothing wrong with this possibility although admittedly it will require many random parameters to reproduce the observed data on $\mathrm{CP}$ violation.

Since the CKM model is simple and seems consistent with known data on CP violation and it would be interesting to see if the above predictive model for neutrinos can coexist with the simple CKM CP violation. One of the objectives of this paper is to study this using the type I seesaw formula. It was mentioned in Ref. 12] that the main reason for the constraint on CP phase being in the third quadrant in the pure type II model has to do with fitting the electron mass and if a nonrenormalizable term is introduced to fix this problem, then the CP phase could be of CKM type.

In this paper, we focus primarily on the same minimal $\mathrm{SO}(10)$ model but with both type I and type II seesaw formula for neutrinos. We obtain the following results: (i) We first show that in a certain limit, one can get the relation $M_{\nu} \simeq c\left(M_{d}-M_{e}\right)$, so that the basic mechanism that led to the maximality of solar and atmospheric neutrino mixings in the case of pure type II seesaw formula is preserved in the type I case. As far as we know, this fact was not noticed in any of the earlier papers that analyzed type I seesaw in minimal SO(10). We then show that the model is in conflict with neutrino data with or without CP violating phases. This is different from the pure type II case where, one could get the neutrino predictions correctly within the renormalizable framework without any CP phase in the Yukawa couplings as well 
as the KM phase in second quadrant. (ii) Secondly, we show that we can restore compatibility with KM CP violation for both type I and type II cases by using the SO(10) model as an effective theory at the GUT scale, where we generate a specific nonrenormalizable term. This not only leads to successful predictions for neutrino mixings but also maintains the CKM model for $\mathrm{CP}$ violation. Of course, the resulting model is not a minimal model anymore since at the Planck scale, it can emerge from a renormalizable theory with two pairs of $\mathbf{1 2 6}$ fields (rather than one in the minimal case). (iii) We also find that the type I model works only for values of $\tan \beta$ higher than 30, providing a way to test the model in supersymmetry experiments. (iv) We evaluate the lepton flavor violating processes and electron electric dipole moment (EDM) by using the predicted leptonic mass matrices in both type I and pure type II case and show that they are in a range accessible to the present experiments. (v) We find that for the type I model to work, intermediate scale $\left(v_{R}\right)$ must be below the GUT scale. We present a brief discussion of unification of gauge couplings in the model to show that this can indeed happen.

This paper is organized as follows: in section 2, we give a basic framework of the minimal $\mathrm{SO}(10)$ model and show that the relation $M_{\nu} \simeq c\left(M_{d}-M_{e}\right)$ needed to understand large neutrino mixings follows in a certain limit even with the type I or mixed type II seesaw. We examine its predictions for the case where the type I seesaw formula is used for discussing neutrino masses and KM phase is kept around 60-70 degrees as required in the standard model fit. We find that the predictions for solar neutrino oscillation do not agree with the current combined data analysis of mixing angles and ratio of mass squared differences for this case. We also discuss the restriction on $\tan \beta$ in the mixed type II and type I models. In section 3 , to remedy the situation of neutrino masses and mixing angles, we include specific types of non-renormalizable terms in the superpotential and show how they are helpful in reconciling the neutrino predictions with CKM CP violation for both type I and type II models. We also discuss the origins of these terms. In sec. 4, we discuss the predictions of the model giving numerical results for various parameters as well as neutrino masses and mixings. In section 5, we discuss the gauge coupling unification in the model and show that the $B-L$ scale required for obtaining neutrino masses is compatible with gauge coupling unification. In sec. 6, we evaluate the leptonic flavor violation and lepton electric dipole moment for both type I and type II models. Section 7 contains our conclusions. 


\section{Minimal Renormalizable SO(10) Model and Its Pre- dictions}

In the minimal $\mathrm{SO}(10)$ model, Yukawa interactions are given as the couplings of the 16dimensional matter spinor $\psi_{i}$ with only one 10-dimensional Higgs $H$ and one $\overline{\mathbf{1 2 6}}$ Higgs $\Delta$. The superpotential for Yukawa interactions is written as

$$
W_{Y}=\frac{1}{2} h_{i j} \psi_{i} \psi_{j} H+\frac{1}{2} f_{i j} \psi_{i} \psi_{j} \Delta
$$

The Yukawa couplings, $h$ and $f$, are complex symmetric $3 \times 3$ matrices.

The SO(10) gauge symmetry breaks down by the Higgs mechanism. There are several breaking pattern in $\mathrm{SO}(10)$ GUT. The $\mathrm{SO}(10)$ symmetry is broken to the left-right group, $G_{2231}=S U(2)_{L} \times S U(2)_{R} \times S U(3)_{c} \times U(1)_{B-L}$, using the $\mathbf{5 4}$ and $\mathbf{2 1 0}$ Higgses. To break $S U(2)_{R} \times U(1)_{B-L}$ down to $U(1)_{Y}$, we will use $\mathbf{1 2 6}+\overline{\mathbf{1 2 6}}$ Higgs multiplets.

The SO(10)-invariant superpotential, Eq.(3), includes MSSM Yukawa couplings plus right handed Majorana mass term which are written by using the MSSM superfields:

$$
W_{Y} \supset Y_{i j}^{u} Q_{i} U_{j}^{c} H_{u}+Y_{i j}^{d} Q_{i} D_{j}^{c} H_{d}+Y_{i j}^{e} L_{i} E_{j}^{c} H_{d}+Y_{i j}^{\nu} L_{i} N_{j}^{c} H_{u}+\frac{1}{2} f_{i j} N_{i}^{c} N_{j}^{c} \Delta_{R},
$$

where $H_{u}$ and $H_{d}$ are MSSM Higgs doublets which are linear combinations of the SM doublets in $\mathbf{1 0}$ and $\overline{\mathbf{1 2 6}}$ Higgs multiplets, and $\Delta_{R}$ is part of the $\overline{\mathbf{1 2 6}}$ field $\Delta$. We note that the VEV of $\Delta_{R}$ gives right handed neutrino masses and breaks $S U(2)_{R} \times U(1)_{B-L}$ symmetry down to $U(1)_{Y}$. As noted the $\mathrm{SO}(10)$ Higgs fields, $H$ and $\Delta$, contain two pairs of $S U(2)_{L}$ Higgs doublets, $H_{10}^{u, d}$ and $H_{126}^{u, d}$, and the MSSM Higgs doublets are linear combinations of two pairs,

$$
\begin{aligned}
& H_{u}=\alpha_{u} H_{10}^{u}+\beta_{u} H_{126}^{u}, \\
& H_{d}=\alpha_{d} H_{10}^{d}+\beta_{d} H_{126}^{d},
\end{aligned}
$$

where $\left|\alpha_{u, d}\right|^{2}+\left|\beta_{u, d}\right|^{2}=1$. The other linear combinations are assumed to be massive around GUT scale. The MSSM Yukawa couplings $Y^{u, d, e, \nu}$ are given by linear combination of $h$ and $f$, and the fermion mass matrices and Majorana mass matrix for right handed neutrino are given $\operatorname{as}^{1}$

$$
\begin{aligned}
& M_{u}=\left(h^{*} \alpha_{u}+f^{*} \beta_{u}\right) v_{u}, \\
& M_{d}=\left(h^{*} \alpha_{d}+f^{*} \beta_{d}\right) v_{d},
\end{aligned}
$$

\footnotetext{
${ }^{1}$ The mass matrices are defined as $-\mathcal{L}_{m}=\overline{\psi_{L}} M \psi_{R}+\frac{1}{2}\left(\nu_{R}\right)^{T} C^{-1} M_{R} \nu_{R}+$ h.c.
} 


$$
\begin{aligned}
M_{e} & =\left(h^{*} \alpha_{d}-3 f^{*} \beta_{d}\right) v_{d}, \\
M_{\nu}^{D} & =\left(h^{*} \alpha_{u}-3 f^{*} \beta_{u}\right) v_{u}, \\
M_{R} & =f^{*} v_{R},
\end{aligned}
$$

where $v_{u, d}$ are VEVs of MSSM Higgs doublets and $v_{R}$ is a VEV of $\Delta_{R}$. We denote that $v_{u}=v \sin \beta$ and $v_{d}=v \cos \beta$, where $v=174 \mathrm{GeV}$. Then we have sumrules for leptonic mass matrices:

$$
\begin{aligned}
M_{e} & =c_{d} M_{d}+c_{u} M_{u}, \\
M_{\nu}^{D} & =\frac{c_{d}+3}{c_{u}}\left(M_{d}-M_{e}\right)+M_{u}, \\
M_{R} & =\frac{M_{d}-M_{e}}{4 \beta_{d} v_{d}} v_{R},
\end{aligned}
$$

where

$$
c_{u}=\frac{4 \cot \beta}{\alpha_{u} / \alpha_{d}-\beta_{u} / \beta_{d}}, \quad c_{d}+3=\frac{-4 \beta_{u} / \beta_{d}}{\alpha_{u} / \alpha_{d}-\beta_{u} / \beta_{d}} .
$$

So if the quark mass matrices $M_{u}$ and $M_{d}$ are our input, then leptonic mass matrices are determined by two complex parameters, $c_{u}$ and $c_{d}$, barring the overall scale and the phase of Majorana neutrino mass matrix, $M_{R}$. Thus, using the masses of three charged leptons, we predict the neutrino mass matrices in terms of only one real parameter. This is an interesting feature of the minimal $\mathrm{SO}(10)$ model and is responsible for the high predictive power of the model.

Let us count the number of parameters in the fermion mass matrices in the minimal $\mathrm{SO}(10)$ model. Rotating the fermion fields by a unitary matrix without violating $\mathrm{SO}(10)$ symmetry (fermions in $\mathbf{1 6}$ are all rotating simultaneously), up-type quark mass matrix, $M_{u}$, can be made real positive diagonal, $M_{u}=\operatorname{diag}\left(m_{u}, m_{c}, m_{t}\right) \equiv D_{u}$. Then down-type quark mass matrix, $M_{d}$, is a general complex symmetric matrix, which has 6 complex parameters. The matrix can be written as $M_{d}=U D_{d} U^{T}$ where $D_{d}$ is real positive diagonal, $D_{d} \equiv \operatorname{diag}\left(m_{d}, m_{s}, m_{b}\right)$, and $U$ is a unitary matrix. The unitary matrix has 9 real parameters ( 3 angles and 6 phases) and we can parameterize the unitary matrix as $U=P_{u}^{*} \bar{U} P_{d}$. The matrices $P_{u}$ and $P_{d}$ are diagonal phase matrices, $P_{u} \equiv \operatorname{diag}\left(e^{i \phi_{u} / 2}, e^{i \phi_{c} / 2}, 1\right)$ and $P_{d} \equiv \operatorname{diag}\left(e^{i \phi_{d} / 2}, e^{i \phi_{s} / 2}, e^{i \phi_{b} / 2}\right)$ and $\bar{U}$ is just same as the Cabibbo-Kobayashi-Maskawa (CKM) matrix, $V_{C K M}$, in which there are 3 mixing angles and 1 phase (KM phase). The phase matrices, $P_{u}$ and $P_{d}$, are unphysical in the MSSM quark sector since they can be absorbed in right handed quark fields, but the phases are relevant parameters in the leptonic mass matrices through the sumrules in Eqs.(12 14). One overall 
phase of $M_{d}$ is irrelevant in the sumrules, using the rotation, $M_{d} \rightarrow e^{i \vartheta} M_{d}, M_{u} \rightarrow M_{u}$. This rotation is generated by $\left(\alpha_{d}, \beta_{d}\right) \rightarrow\left(e^{i \vartheta} \alpha_{d}, e^{i \vartheta} \beta_{d}\right)$. So, we can choose the phase $\phi_{b}$ to be zero. Finally, we have 19 parameters in the sumrules: 14 real parameters in quark mass matrices $M_{u}$ and $M_{d}$ (6 quark masses, 3 mixing angles, $1 \mathrm{KM}$ phase, and 4 unknown phases, $\left.\phi_{u, c, d, s}\right), 2$ complex parameters, $c_{u}$ and $c_{d}$, and 1 overall scale parameter in the Majorana mass matrix $M_{R}$ (overall phase of $M_{R}$ is not counted since it is not physical). On the other hand, the MSSM Yukawa couplings plus the right handed Majorana mass in Eq.(44), give us 32 parameters: 10 parameters in $Y^{u}$ and $Y^{d}$ (6 quark masses and 3 mixings and $1 \mathrm{KM}$ phase), 10 parameters in $Y^{e}$ and $Y^{\nu}$ in the same way, and then 12 parameters in the Majorana mass matrix which is generally a symmetric matrix. So, in the minimal SO(10) model, we have less parameters than the MSSM.

The quark masses, mixings and the KM phase are our inputs. We can redefine the basis of fermions, and the up- and down-type quark mass matrices are written as $M_{u}=D_{u} P_{u}^{2}$, $M_{d}=V_{C K M} D_{d} P_{d}^{2} V_{C K M}^{T}$. The authors in Ref.[7] assumed that the phase matrices $P_{u, d}^{2}$ are real and they found a solution to generate the observed quark masses and the CKM matrix and the charged lepton masses in the case where $\phi_{d, s, c}=\pi$ and $\phi_{u}=0, \pi$. In our general analysis, we take those phases $\phi_{d, s, u, c}$ to be arbitrary. Those phases are constrained to obtain the bi-maximal neutrino oscillation data.

Let us see how one can obtain a numerical fit of the parameters in the lepton sector at the GUT scale. The charged lepton mass matrix is written as $M_{e}=c_{d} M_{d}+c_{u} M_{u}$. So, $\tau$ and $\mu$ masses are approximately (neglecting generation mixing and phases),

$$
\pm m_{\tau} \simeq c_{d} m_{b}+c_{u} m_{t}, \quad \pm m_{\mu} \simeq c_{d} m_{s}+c_{u} m_{c}
$$

Using the relations $m_{c} / m_{t} \ll m_{s} / m_{b}$ and $m_{\tau} \simeq m_{b}$, we obtain $c_{d} \simeq \pm m_{\mu} / m_{s}$ and $c_{u} \simeq$ $-m_{b} / m_{t}\left(c_{d} \pm 1\right)$, and thus $\left|c_{d}\right|$ is about 3, naively. In this naive approximation, however, the electron mass becomes about $c_{d} m_{d}$, which is too large, so we cannot neglect the off-diagonal elements, and we need a fine-tuning in $\operatorname{det}\left(M_{d}+\kappa M_{u}\right)$, where $\kappa \equiv c_{u} / c_{d}$, by a suitable choice of $\kappa$. The numerical fit is given in Ref. [7]. The key ingredient to fit the electron mass is the strange quark mass. Actually, the current strange quark mass still has large experimental error and we can make it to be a parameter in the model. We show the values of the strange quark mass (at $1 \mathrm{GeV}$ ) and $\left|c_{d}\right|$ by varying the KM phase in Fig.1. We take the KM phase to be in the first and second quadrant without loss of generality, since the sumrule does not change under the conjugation. The three real parameters are consumed to fix the three charged lepton 

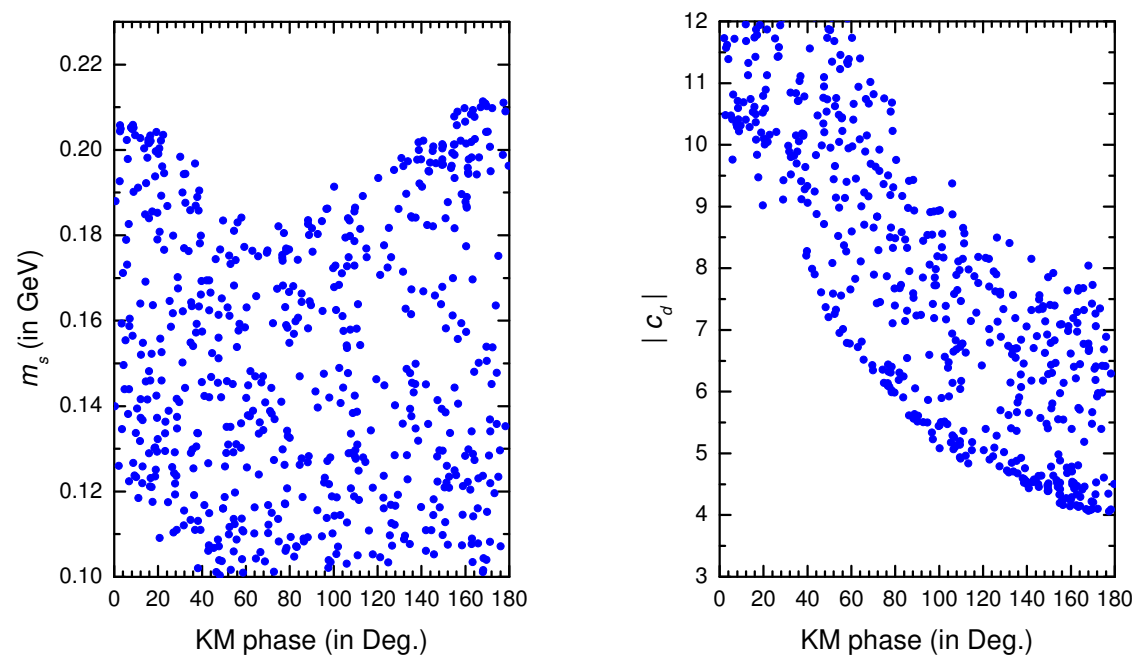

Figure 1: The strange quark mass (at $1 \mathrm{GeV}$ ) and $\left|c_{d}\right|$ to fit charged lepton masses are shown.

masses: $\kappa$ and $\left|c_{d}\right|$ are determined and the phase of $c_{d}$ is still undetermined.

We now determine the neutrino mass matrix. First, in the pure-type II case, the light neutrino mass, $\mathcal{M}_{\nu}^{\mathrm{II}}$, is given as $f^{*} v_{L}$ which is proportional to $M_{d}-M_{e}$. The charged-lepton mass matrix is written as $M_{e}=c_{d}\left(M_{d}+\kappa M_{u}\right)$. We define the complex number $\xi$ as

$$
\xi \equiv\left|c_{d}\right|\left(M_{d}+\kappa M_{u}\right)_{33} / m_{\tau} .
$$

By definition, $|\xi| \simeq 1$. The phase of $\xi$ is determined by the charged lepton mass fitting. The $(3,3)$ element of $M_{e}$ is then $\xi e^{i \sigma} m_{\tau}$, where $\sigma$ is a phase of $c_{d}, e^{i \sigma}=c_{d} /\left|c_{d}\right|$. We denote $\hat{\xi} \equiv \xi e^{i \sigma}$. The pure type II neutrino mass matrix is given as

$$
\begin{aligned}
\mathcal{M}_{\nu}^{\mathrm{II}} & \propto\left(1-c_{d}\right) M_{d}-c_{u} M_{u} \\
& \simeq\left(1-c_{d}\right)\left(\begin{array}{ccc}
m_{d} e^{i \phi_{d}}+V_{u s}^{2} m_{s} e^{i \phi_{s}} & V_{u s} m_{s} e^{i \phi_{s}} & V_{u b} m_{b} \\
V_{u s} m_{s} e^{i \phi_{s}} & m_{s} e^{i \phi_{s}} & V_{c b} m_{b} \\
V_{u b} m_{b} & V_{c b} m_{b} & \left(m_{b}-\hat{\xi} m_{\tau}\right) /\left(1-c_{d}\right)
\end{array}\right) .
\end{aligned}
$$

In the expression, $c_{u} m_{c}$ and $c_{u} m_{u}$ terms in the diagonal element are neglected. Choosing the phase $\sigma$ to make $\hat{\xi} \simeq 1$, the $(3,3)$ entry of the matrix is $m_{b}-m_{\tau}$. Since $b-\tau$ mass convergence implies that $m_{b}$ and $m_{\tau}$ become close to each other as we move to the GUT scale, we can assume that $m_{b}-m_{\tau} \sim\left(1-c_{d}\right) V_{c b} m_{b}$, which implies that the neutrino mass matrix is given as

$$
\mathcal{M}_{\nu} \sim\left(\begin{array}{ccc}
\lambda^{2} & \lambda & \lambda \\
\lambda & 1 & 1 \\
\lambda & 1 & 1
\end{array}\right) m_{0}
$$


where $\lambda \simeq V_{u s} \sim 0.2$. We then obtain bi-large neutrino mixing: both atmospheric and solar angle are large. The third neutrino mass, $m_{\nu_{3}}$, is about $m_{0}$. To obtain the large solar mixing, determinant of (2-3) block of the neutrino matrix is required to be less than $\lambda m_{0}^{2}$, and then the second neutrino mass, $m_{\nu_{2}}$, is about $\lambda m_{0}$. So the mass squared ratio is predicted as $\Delta m_{\odot}^{2} / \Delta m_{A}^{2} \sim \lambda^{2}$. The 1-3 neutrino mixing is also predicted as $U_{e 3} \sim \lambda$.

Next we consider the case of mixed type II neutrino mass matrix. This case is more complicated than the pure type II case. From Eqs.(13) 14), the mixed type II seesaw matrix is given as

$$
\mathcal{M}_{\nu}^{\mathrm{II} \text { mixed }} \propto\left(M_{d}-M_{e}\right)(1+\Delta)+2 \frac{c_{u}}{c_{d}+3} M_{u}+\left(\frac{c_{u}}{c_{d}+3}\right)^{2} M_{u}\left(M_{d}-M_{e}\right)^{-1} M_{u}
$$

The first term is just the same as in the pure type II case. When $\Delta=0$, we get the type I case. The second and third terms might give a hierarchical structure to the (2-3) block of neutrino mass matrix, and would spoil large atmospheric mixing. The $(3,3)$ element of the Eq.(20) can be written approximately as

$$
\left((1-\hat{\xi})(1+\Delta)+2 \frac{\hat{\xi}-c_{d}}{c_{d}+3}+\zeta\left(\frac{\hat{\xi}-c_{d}}{c_{d}+3}\right)^{2}\right) m_{\tau}
$$

where $\zeta=\left[\left(M_{d}-M_{e}\right)^{-1}\right]_{33} m_{\tau}$. This element is of the order of $m_{\tau}$ or larger in general. However, if the $(3,3)$ element can be canceled to the order of $(1+\Delta)\left(1-c_{d}\right) V_{c b} m_{\tau}$, then the bi-large mixing neutrino matrix can be realized. Assuming that $\hat{\xi} \simeq 1$ as in pure type II case, the cancellation condition of the second and third terms is $c_{d}=-(6+\zeta) /(2-\zeta)$. In our numerical analysis, the parameter $\zeta$ is almost real and $\zeta \simeq 1$. Thus, the cancellation condition is $c_{d} \sim-7$. At that time, the phase of $c_{d}$ is almost $\pi$, and then the phase of $\xi$ must be almost $\pi$ from the condition $\hat{\xi} \simeq 1$. The condition can be satisfied if the strange quark has a smaller value of mass, since $\left|c_{d}\right| \sim m_{\mu} / m_{s}$ naively. According to our numerical analysis, $\hat{\xi}$ is not necessary to be 1 and $c_{d}$ can be different values from -7 . However, $\left|c_{d}\right|$ has a lower bound, $\left|c_{d}\right| \gtrsim 5$ to cancel the $(3,3)$ element of the neutrino mass matrix in our analysis. Consequently, smaller value of strange mass is favored in the mixed type II case.

In both pure and mixed type II (and of course in the type I case), the neutrino mass matrix structure in Eq.(19) which gives bi-large mixing can be obtained. Then we have a good prediction of the 1-3 mixing angle, $\left|U_{e 3}\right| \simeq \lambda$. Actually, in the numerical studies in Ref. 12] (type II) and Ref. [8] (type I), the prediction of $\left|U_{e 3}\right|$ is about 0.17-0.18. Another important prediction of the minimal SO(10) model is the solar mixing angle and the ratio of mass squared differences, $\Delta m_{\odot}^{2} / \Delta m_{A}^{2}$. The predictions are almost determined by the (2-3) block of the matrix. The (2-3) 
block is approximately written as

$$
\mathcal{M}_{\nu}^{(2-3)} \propto\left(\begin{array}{cc}
m_{s} e^{i \phi_{s}} & V_{c b} m_{b} \\
V_{c b} m_{b} & \epsilon m_{\tau}
\end{array}\right)
$$

where $\epsilon$ is a cancellation factor, $\epsilon m_{\tau} \simeq\left(M_{d}-M_{e}\right)_{33} /\left(1-c_{d}\right)$ in the pure type II case, and $\epsilon m_{\tau}$ is approximately Eq.(21) divided by $1-c_{d}$ in the mixed type II case. The $|\epsilon|$ should be less than $V_{c b} \sim \lambda^{2}$ to give rise to a large atmospheric mixing. The condition to obtain a large maximal mixing angle is that the determinant of the (2-3) block is canceled,

$$
\left|\epsilon e^{i \phi_{s}} m_{s} m_{\tau}-V_{c b}^{2} m_{b}^{2}\right| \lesssim O(\lambda) V_{c b}^{2} m_{b}^{2}
$$

This condition also provides a small mass differences squared ratio, $\Delta m_{\odot}^{2} / \Delta m_{A}^{2} \sim \lambda^{2}$. To satisfy the condition, one needs $\epsilon e^{i \phi_{s}} \simeq+|\epsilon|$, and $|\epsilon|$ can not be very small. Then, maximal atmospheric mixing requires a relation $m_{s}-|\epsilon| m_{\tau} \ll 4 V_{c b} m_{b}$. Assuming $m_{s} \simeq|\epsilon| m_{\tau}$, we obtain the condition for the bi-maximal neutrino mixing and the small mass squared ratio as

$$
m_{s} \simeq V_{c b} m_{b}
$$

From the experimental data, the strange/bottom mass ratio at the GUT scale is always smaller than $V_{c b}$, thus a larger strange mass gives rise to larger mixing angles in the minimal $\mathrm{SO}(10)$ model. Furthermore, in the minimal model, the strange quark mass is constrained due to electron mass fitting. As we can see in Fig.1, the maximal values of the strange mass depend on the KM phase, and the second quadrant KM phase gives larger strange quark mass. As a result, the bi-maximal neutrino mixings and the small mass squared ratio favor the second quadrant KM phase. This result is in agreement with the numerical studies in Ref. 12. In the case of mixed type II or type I, smaller values of strange quark mass are favored to obtain the cancellation of the $(3,3)$ component of the neutrino mass matrix as we have noted. So in type I and mixed type II case, there is a tension between getting large neutrino mixings in general and getting their precise values as well as the desired mass square ratio. As a result, the minimal $\mathrm{SO}(10)$ model with type I or mixed type II predicts that the solar mixing angle is not very large and the mass squared ratio is not very small as long as the atmospheric neutrino mixing is maximal. We give our numerical data plotting for the solar mixing angle and mass squared ratio in the Fig 2. In the mixed type II or type I case, the mixing angle and mass squared ratio are bounded for any KM phase, and recent data fitting excludes such bounds more than $2 \sigma$ level [2]. 


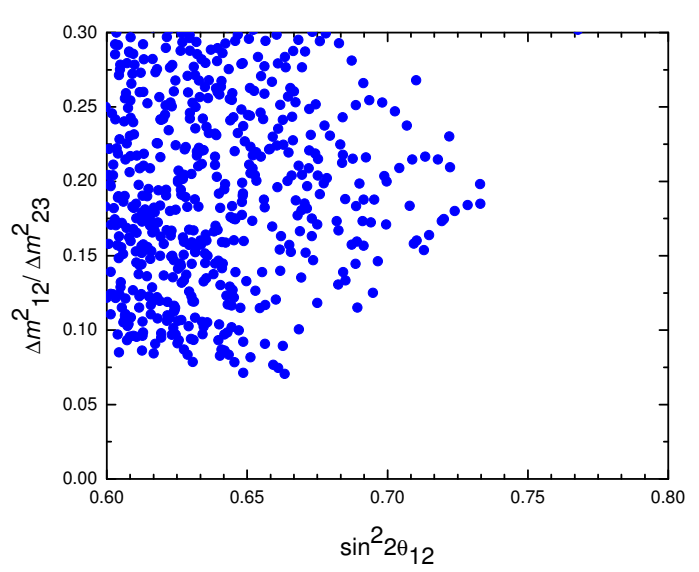

Figure 2: The solar mixing angle and ratio of mass squared differences are dot-plotted for $\sin ^{2} 2 \theta_{23}>0.89$. The allowed region from recent data fitting lies in the right-bottom corner in the figure.

Next we will see the $\tan \beta$ bound in the minimal SO(10) model. From Eq.(15), we obtain

$$
\frac{\alpha_{u}}{\alpha_{d}}=\frac{1-c_{d}}{c_{u}} \cot \beta, \quad \frac{\beta_{u}}{\beta_{d}}=-\frac{c_{d}+3}{c_{u}} \cot \beta .
$$

Since $\alpha_{u, d}$ and $\beta_{u, d}$ are Higgs mixings, we have unitarity constraint $\left|\alpha_{u, d}\right|^{2}+\left|\beta_{u, d}\right|^{2}=1$. Thus we have $\left(\left|\alpha_{u} / \alpha_{d}\right|-1\right)\left(\left|\beta_{u} / \beta_{d}\right|-1\right) \leq 0$, and

$$
\min \left(\left|\frac{c_{d}+3}{c_{u}}\right|,\left|\frac{1-c_{d}}{c_{u}}\right|\right) \leq \tan \beta \leq \max \left(\left|\frac{c_{d}+3}{c_{u}}\right|,\left|\frac{1-c_{d}}{c_{u}}\right|\right) .
$$

In the case where $\hat{\xi} \simeq 1$ which is favored to obtain bi-large structure, $\left(1-c_{d}\right) / c_{u} \simeq m_{t} / m_{b}$, and then we have lower bounds of $\tan \beta$, such as $\tan \beta \gtrsim m_{t} / m_{b}\left|\left(c_{d}+3\right) /\left(1-c_{d}\right)\right|$. In the case where $c_{d} \sim-7$ which is favored in the mixed type II (and type I) case, the lower bound of $\tan \beta$ is roughly 30. To obtain small $\tan \beta$ such as $\tan \beta \sim 10, c_{d} \sim-3.5$ is needed. Such small $\left|c_{d}\right|$ can happen only in the case where the KM phase is close to 180 degree in the minimal $\mathrm{SO}(10)$ model.

In the type I seesaw case, the scale of the right handed Majorana mass is predictable. Using the Eqs.(13),14), the light neutrino mass scale, $m_{0}$ in Eq.(19), can be written as

$$
m_{0} \simeq\left(1-c_{d}\right)\left(\frac{c_{d}+3}{c_{u}}\right)^{2} V_{c b} m_{b} \frac{4 \beta_{d} v_{d}}{v_{R}}
$$

So using the mass squared magnitude from the atmospheric neutrino data, we can fix the mass parameter, $m_{0}$, and obtain the right handed Majorana mass matrix, Eq.(14). The lightest 
Majorana mass, which is an important scale for leptogenesis, is about $10^{11} \mathrm{GeV}$. Also we obtain the upper bound of $v_{R}$ since $\left|\beta_{d}\right|$ is less than 1 .

Now let us summarize the predictions of the minimal $\mathrm{SO}(10)$ model. In both pure type II and mixed type II, we can obtain the bi-large mixing structure, Eq.(19), for the solar and the atmospheric oscillation. In the bi-large neutrino matrix, the 1-3 mixing is well predicted as $\left|U_{e 3}\right| \simeq 0.17-0.18$. In the mixed type II (and type I) case, larger $\left|c_{d}\right|$ is favored to obtain bi-large structure in the neutrino mass matrix. In order to obtain both bi-maximal neutrino mixing and small mass squared ratio, a larger strange quark mass is favored, and this requires a smaller $\left|c_{d}\right|$. So, in the mixed type II (and type I) case, the bi-maximal neutrino and the small mass squared ratio are not favored, and it is hard to achieve a best fit value of the recent combined data analysis. In the pure type II case, the condition of bi-large mixing is just $\hat{\xi} \simeq 1$, and $\left|c_{d}\right|$ is not constrained. However, due to the electron mass fitting, the strange mass is related to the KM phase, and if the KM phase is 60-80 degree, the strange mass can not be large and thus it is hard to achieve the bi-maximal mixing and the small mass squared ratio in the pure type II case as well. If the KM phase is in the second quadrant and especially for the larger value of KM phase, the strange quark mass can be large and the best fit values for neutrino oscillation can be achieved. The $\tan \beta$ in the mixed type II and type I model is greater than 30 roughly and the $B-L$ breaking scale has an upper-bound in the type I model.

\section{Inclusion of Nonrenormalizable terms}

As we have seen in the previous section, the fitting of three charged-lepton masses (especially, electron mass) gives relatively small strange quark mass in the case where the KM phase is 60-80 degree. Then the $(2,2)$ element of the neutrino mass matrix is relatively smaller than $(2,3)$ element, and as a result, we get bounds for the solar mixing angle and the mass squared ratio. In this section, in order to reproduce the CKM model of $\mathrm{CP}$ violation at low energies, we employ corrections to the $\mathrm{SO}(10)$ model which originate from non-renormalizable terms in the superpotential.

The most simple non-renormalizable term is written including an extra singlet $S$

$$
W_{Y}^{\mathrm{nr}}=\frac{1}{2} \frac{S}{M_{P}}\left(h_{i j}^{\prime} \psi_{i} \psi_{j} H_{10}+f_{i j}^{\prime} \psi_{i} \psi_{j} H_{126}\right) .
$$

In this case, there is no change from the results of the renormalizable case since the effect of these terms is simply to redefine the couplings $h$ and $f$ of Eq.(3). We therefore seek other renormalizable terms. 
We will employ 210 Higgs multiplet to remedy the situation. Note that $\mathbf{2 1 0}$ Higgs (to be denoted $\Sigma$ ) can be used to break the $\mathrm{SO}(10)$ symmetry down to $S U(3)_{c} \times S U(2)_{L} \times S U(2)_{R} \times$ $U(1)_{B-L}$ if we give the $(\mathbf{1}, \mathbf{1}, \mathbf{1})$ and $(\mathbf{1}, \mathbf{1}, \mathbf{1 5})$ (under $\left.G_{224}\right)$ submultiplets of $\mathbf{2 1 0}$ vev. One can then include the term

$$
\frac{1}{2} \frac{1}{M_{P}} h_{i j}^{\prime} \psi_{i} \psi_{j} H \Sigma
$$

Since $\Sigma$ has four $\mathrm{SO}(10)$ indices, the combination $H \Sigma$ that can couple to spinors is either a $\mathbf{1 2 0}$ or 126 representation. We restrict the high scale theory (ultraviolet completion) from which the nonrenormalizable term originates in such a way that no 120 term in the effective $H \Sigma$ term couples to matter. For instance, we could add a second $\mathbf{1 2 6}+\overline{\mathbf{1 2 6}}$ pair of field with a high mass $M \gg M_{U}$ which does not develop a VEV with a superpotential given as follows ${ }^{2}$ :

$$
W^{\prime}=h^{\prime} \psi \psi \bar{\Delta}^{\prime}+M \bar{\Delta}^{\prime} \Delta^{\prime}+\Delta^{\prime} \Sigma H
$$

The effective theory below the scale $M$ has a nonrenormalizable term of the type in Eq. (30) where only 126 part of $\Sigma H$ field product contributes. If only $(\mathbf{1}, \mathbf{1}, \mathbf{1})$ and $(\mathbf{1}, \mathbf{1}, \mathbf{1 5})$ submultiplets of $\mathbf{2 1 0}$ acquire vev, the effective operator that contributes to fermion masses then has $(2,2,15)$ quantum numbers under $G_{224}$. This leads to mass formulae for the quarks and leptons of the following type.

$$
\begin{aligned}
M_{u} & =\left(h^{*} \alpha_{u}+f^{*} \beta_{u}\right) v_{u}+h^{*} \alpha_{u} v_{u}\langle\Sigma\rangle / M_{P}, \\
M_{d} & =\left(h^{*} \alpha_{d}+f^{*} \beta_{d}\right) v_{d}+h^{\prime *} \alpha_{d} v_{d}\langle\Sigma\rangle / M_{P}, \\
M_{e} & =\left(h^{*} \alpha_{d}-3 f^{*} \beta_{d}\right) v_{d}-3 h^{*} \alpha_{d} v_{d}\langle\Sigma\rangle / M_{P}, \\
M_{\nu}^{D} & =\left(h^{*} \alpha_{u}-3 f^{*} \beta_{u}\right) v_{u}-3 h^{\prime *} \alpha_{u} v_{u}\langle\Sigma\rangle / M_{P}, \\
M_{R} & =f^{*} v_{R},
\end{aligned}
$$

where $v_{u, d}$ are VEVs of MSSM Higgs doublets and $v_{R}$ is VEV of $\Delta_{R}$. Note also that $h^{\prime}$ is not a symmetric matrix in general, but we assume that $h^{\prime}$ is a symmetric matrix like $h$ and $f$ for simplicity.

We can now rewrite the above equations as follows

$$
\begin{aligned}
& M_{e}=M_{e}^{0}+\Delta M_{e}, \\
& M_{\nu}^{D}=\frac{c_{d}+3}{c_{u}}\left(M_{d}-M_{e}^{0}\right)+M_{u}+\frac{1-c_{d}}{c_{u}} \Delta M_{e}, \\
& M_{R}=\frac{M_{d}-M_{e}^{0}}{4 \beta_{d} v_{d}} v_{R},
\end{aligned}
$$

\footnotetext{
${ }^{2}$ For another nonminimal version with multiple $\mathbf{1 2 6}$ at the GUT scale, see Ref.[15].
} 
where $M_{e}^{0}=c_{d} M_{d}+c_{u} M_{u}$ and $\Delta M_{e}=-4 h^{\prime *} \alpha_{d} v_{d}\langle\Sigma\rangle / M_{P}$. In this sumrules, we can break the relation of the $(2,2)$ element between charged-lepton and neutrino mass matrices, and thus we can increase $(2,2)$ element of seesaw neutrino mass matrix. In the mixed type II (and type I) case, we have to increase the $(2,2)$ element while keeping $\left|c_{d}\right|$ to be larger than 5 . In fact, we find a large solar mixing solution in type I when we switch on the $(2,2)$ element of the $\Delta M_{e}$.

In the pure type II case, we do not need the $(2,2)$ element of $\Delta M_{e}$ since a small $\left|c_{d}\right|$ can give rise to maximal mixings. Instead, we need the $(1,1)$ element to cancel and to give the desired electron mass and we then find a large solar mixing solution in the case where KM phase is in the first quadrant.

\section{Numerical Results}

As already noted, the minimal SO(10) model is predictive because fermions have only two Yukawa couplings, one with 10 and one $\overline{\mathbf{1 2 6}}$ Higgses. This leads to a sumrule for the leptonic mass matrices which determine the the Dirac neutrino and right handed Majorana mass matrices from the observed neutrino data. In a generic model for neutrino masses, the neutrino oscillation data gives us information about the neutrino masses and mixing angles of light neutrino mass matrix, but no information about the Dirac neutrino and the right handed Majorana mass matrices separately. On the other hand, a separate information of the Dirac neutrino and the right handed Majorana mass matrices are important to extract the predictions of the models for lepton flavor violation and leptonic CP violation. In the minimal SO(10) model however, these matrices are completely determined.

In type I case that we are considering in this paper, we get:

$$
Y_{\nu}=\left(\begin{array}{ccc}
-0.00159-0.00014 i & 0.00067-0.0036 i & 0.017+0.015 i \\
0.00369-0.00026 i & 0.0182+0.0107 i & -0.046-0.0228 i \\
-0.022+0.0085 i & -0.02-0.0477 i & 0.58+0.126 i
\end{array}\right)
$$

and

$$
M_{R}=\left(\begin{array}{ccc}
0.00059+0.000048 i & -0.00022+0.0012 i & -0.0058-0.0051 i \\
-0.00022+0.0012 i & -0.0014-0.0063 i & 0.011-0.013 i \\
-0.0058-0.0051 i & 0.011-0.013 i & -0.037-0.0086 i
\end{array}\right) \times 10^{14.08} \mathrm{GeV}
$$

The above matrices are calculated for $\tan \beta=40$ and are in the basis where the charged lepton

masses are diagonal. The scale $10^{14.08} \mathrm{GeV}$ is the VEV of $\overline{\mathbf{1 2 6}}$ Higgs which couples to fermions and this magnitude of the scale is the maximum possible value given the inputs of the quark 
masses and the CKM mixings. The $h$ and $f$ in the basis where $M_{u}$ diagonal are given as follows:

$$
\begin{gathered}
h^{*}=\left(\begin{array}{ccc}
0.0012-0.0002 i & 0.00067-0.00014 i & 0.0011+0.0035 i \\
0.00067-0.00014 i & -0.0029-0.00122 i & -0.037+0.0043 i \\
0.0011+0.0035 i & -0.037+0.0043 i & 1.15+0.129 i
\end{array}\right), \\
f^{*}=\left(\begin{array}{ccc}
-0.0012-0.00006 i & -0.0025+0.000062 i & 0.00088-0.0021 i \\
-0.0025+0.000062 i & -0.0097+0.00061 i & 0.021-0.0011 i \\
0.00088-0.0021 i & 0.021-0.0011 i & -0.0012-0.0345 i
\end{array}\right) .
\end{gathered}
$$

The neutrino mixing angles and mass squared ratio are given as:

$$
\sin ^{2} \theta_{12}=0.87, \sin ^{2} \theta_{23}=0.92,\left|U_{e 3}\right|=0.22, \Delta m_{\odot}^{2} / \Delta m_{A}^{2}=0.051
$$

At $\tan \beta=50$, the same matrices are given by:

$$
Y_{\nu}=\left(\begin{array}{ccc}
-0.0017-0.0004 i & 0.0012-0.0039 i & 0.016+0.019 i \\
0.004-0.00056 i & 0.0194+0.011 i & -0.053-0.023 i \\
-0.023+0.01 i & -0.025-0.052 i & 0.660+0.13 i
\end{array}\right)
$$

and

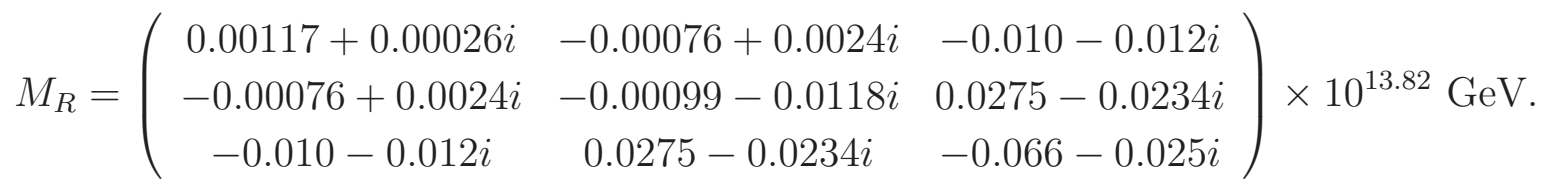

The neutrino mixing angles and mass squared ratio are given as:

$$
\sin ^{2} \theta_{12}=0.81, \sin ^{2} \theta_{23}=0.90,\left|U_{e 3}\right|=0.22, \Delta m_{\odot}^{2} / \Delta m_{A}^{2}=0.06
$$

In the pure type II case, we get :

$$
Y_{\nu}=\left(\begin{array}{ccc}
0.0008-0.001 i & -0.001-0.0057 i & 0.004-0.026 i \\
0.003+0.0013 i & -0.0056+0.00015 i & -0.053-0.008 i \\
-0.000039+0.0014 i & -0.012+0.00096 i & 0.627+0.1019 i
\end{array}\right)
$$

and

$$
M_{R}=\left(\begin{array}{ccc}
-0.0016-0.000047 i & -0.0036-0.000029 i & 0.00077+0.0028 i \\
-0.0036-0.000029 i & -0.014-0.00044 i & 0.026+0.0008 i \\
0.00077+0.0028 i & 0.026+0.0008 i & -0.0158-0.0078 i
\end{array}\right) \times 10^{16.24} \mathrm{GeV}
$$

The above matrices are calculated for $\tan \beta=50$ and are in the basis where the charged lepton masses are diagonal. The scale $10^{16.24} \mathrm{GeV}$ is the scale where $\mathrm{SO}(10)$ gets broken to $G_{2231}$ which 
subsequently breaks down to the SM. The neutrino mixing angles and mass squared ratio are given as:

$$
\sin ^{2} \theta_{12}=0.85, \sin ^{2} \theta_{23}=0.91,\left|U_{e 3}\right|=0.22, \Delta m_{\odot}^{2} / \Delta m_{A}^{2}=0.084
$$

At $\tan \beta=40$, the same matrices are:

$$
Y_{\nu}=\left(\begin{array}{ccc}
-0.00027-0.00066 i & -0.0046+0.00089 i & -0.023-0.0023 i \\
-0.00073+0.0028 i & 0.00074-0.0037 i & 0.007-0.052 i \\
-0.0008-0.0001 i & 0.0013-0.0079 i & -0.103+0.57 i
\end{array}\right)
$$

and

$$
M_{R}=\left(\begin{array}{ccc}
-0.00078+1.5 \cdot 10^{-6} i & -0.0018+0.00040 i & 0.00039+0.0012 i \\
-0.0018+0.000040 i & -0.007-8.7 \cdot 10^{-6} i & 0.012+7.8 \cdot 10^{-6} i \\
0.00039+0.0012 i & 0.012+7.8 \cdot 10^{-6} i & -0.0081-0.00013 i
\end{array}\right) \times 10^{16.24} \mathrm{GeV}
$$

The neutrino mixing angles and mass squared ratio are given as:

$$
\sin ^{2} \theta_{12}=0.85, \sin ^{2} \theta_{23}=0.92,\left|U_{e 3}\right|=0.20, \Delta m_{\odot}^{2} / \Delta m_{A}^{2}=0.058
$$

Using these fits, we will calculate the lepton flavor violating processes and the amount of leptonic CP violation in type I and pure type II scenarios in the mSUGRA model in sec. 6.

Even though all the examples we have given have large $U_{e 3}$, its value can be much smaller due to the presence of new parameters in the higher dimensional term.

\section{Gauge Coupling Unification}

From the results of the previous section (Eq. (40) and (45)), we see that for the type I model to be successful in predicting neutrino masses, the intermediate scale $\left(v_{R}\right)$ must be below the GUT scale and around $10^{14} \mathrm{GeV}$ for our examples. We assume the resulting symmetry breaking chain is of type, $S O(10) \rightarrow S U(3)_{c} \times S U(2)_{L} \times S U(2)_{R} \times U(1)_{B-L} \rightarrow S U(3)_{c} \times S U(2)_{L} \times U(1)_{Y}$. This is very different from the gauge coupling unification scenario in MSSM. It is therefore important check if the type I models are compatible with gauge coupling unification.

In our model, in the scale region $M_{S U S Y} \leq \mu \leq v_{R}$, the spectrum is that of familiar MSSM. Above the $v_{R}$ scale, the symmetry group expands to $S U(3)_{c} \times S U(2)_{L} \times S U(2)_{R} \times U(1)_{B-L}$. In addition to the new gauge bosons associated with this new symmetry, the new matter and Higgs that contribute are the following: three $\mathrm{RH}$ neutrinos as part of the $S U(2)_{R}$ lepton doublet; one bidoublet from which the MSSM doublets emerged. In addition we need either the $B-L=2$ 


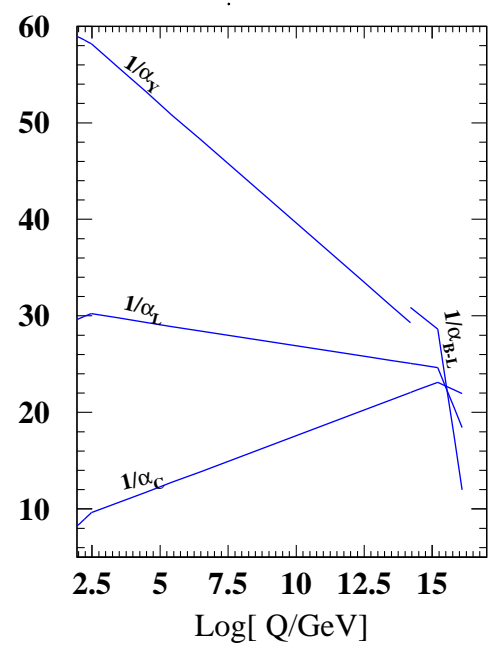

Figure 3: The couplings $1 / \alpha_{i}$ are plotted as a function of scale.

triplet pair $\left(\Delta_{L}+\bar{\Delta}_{L} \oplus \Delta_{R}+\bar{\Delta}_{R}\right)$ and $x[(\mathbf{3}, \mathbf{3}, \mathbf{1},-2 / 3)+(\overline{\mathbf{3}}, \mathbf{3}, \mathbf{1}, 2 / 3)] \oplus y[(\mathbf{3}, \mathbf{1}, \mathbf{3},-2 / 3)+$ $(\overline{\mathbf{3}}, \mathbf{3}, \mathbf{1}, 2 / 3)$ to have successful gauge coupling unification. If we use the Higgs spectrum from Ref. [16] in the context of our model, we find that $x \oplus y$ (from 210) and $\Delta_{L}+\bar{\Delta}_{L} \oplus \Delta_{R}+\bar{\Delta}_{R}$ are at about the $v_{R}$ scale. Using these fields, the Fig. 3 shows the coupling unification in this model and the gauge unification happens at about $10^{15.5} \mathrm{GeV}$. We have chosen the $x, y$ and $\Delta$ Higgs masses to be 6 times the $v_{R}$ scale to get this value.

First of all it is gratifying that the type I model is compatible with gauge coupling unification with the desired value of $v_{R}$ scale. Furthermore the lowering of the ultimate unification scale implies that the gauge exchange contributions to the proton decay in this model lead to proton lifetimes much lower than the MSSM unifying without an intermediate scale and will be around the current lower limit $5 \times 10^{33}$ yrs when we include the threshold corrections [17. The profile of the proton decay modes in the type I case will be substantially different from the pure type II case recently discussed [18] and is presently under investigation.

\section{Lepton Flavor Violating Processes and Lepton Edm}

\subsection{Lepton Flavor Violation}

We now discuss the lepton flavor violating processes e.g. $\mu \rightarrow e \gamma, \tau \rightarrow \mu \gamma$ etc. The operator for $l_{i} \rightarrow l_{j}+\gamma$ is:

$$
\mathcal{L}_{l_{i} \rightarrow l_{j} \gamma}=\frac{i e}{2 m_{l}} \overline{l_{j}} \sigma^{\mu \nu} q_{\nu}\left(a_{l} P_{L}+a_{r} P_{R}\right) l_{i} \cdot A_{\mu}+\text { h.c. }
$$


where $P_{L, R} \equiv\left(1 \mp \gamma_{5}\right) / 2$ and $\sigma^{\mu \nu} \equiv \frac{i}{2}\left[\gamma^{\mu}, \gamma^{\nu}\right]$. The decay width for $l_{i} \rightarrow l_{j}+\gamma$ can be written as:

$$
\Gamma\left(l_{i} \rightarrow l_{j}+\gamma\right)=\frac{m_{\mu} e^{2}}{64 \pi}\left(\left|a_{l}\right|^{2}+\left|a_{r}\right|^{2}\right)
$$

Then the branching ratio is obtained by multiplying this decay width with the life time of the $l_{i}$ lepton. The supersymmetric contributions include the neutralino and chargino diagrams [19].

We work in the basis where the charged lepton masses are diagonal at the highest scale of the theory. We first start with pure type II seesaw model. We assume that $v_{R}$ (the scale where $G_{2231}$ breaks down to SM) is at the GUT scale. So we have just MSSM and right handed neutrinos below the GUT scale. The right handed masses have hierarchies and therefore get decoupled at different scales. The flavor-violating pieces present in $Y_{\nu}$ induces flavor violations into the charged lepton couplings and into the soft SUSY breaking masses e.g. $m^{2}$ terms etc. through the following RGEs:

$$
\begin{aligned}
d Y_{e} / d t & =\frac{1}{16 \pi^{2}}\left(Y_{\nu} Y_{\nu}^{\dagger}+\cdots\right) Y_{e} \\
d m_{L L}^{2} / d t & =\frac{1}{16 \pi^{2}}\left(Y_{\nu} Y_{\nu}^{\dagger} m_{L L}^{2}+m_{L L}^{2} Y_{\nu} Y_{\nu}^{\dagger}+\cdots\right)
\end{aligned}
$$

We use the mSUGRA universal boundary conditions at the GUT scale to draw the figures. In Figs. 4,6] we show the $\operatorname{BR}[\mu \rightarrow e \gamma]$ and $\operatorname{BR}[\tau \rightarrow \mu \gamma]$ as a function of $m_{1 / 2}$ for different values of $A_{0}$. The lightest neutralino is the dark matter candidate in this model and we satisfy the $2 \sigma$ range of the recent relic density constraint $\Omega_{\mathrm{CDM}}=0.1126_{-0.009}^{+0.008}[20$ in the parameter space. When we satisfy the relic density constraint, the $m_{0}$ gets determined. For example, $m_{0}$ varies between $60-100 \mathrm{GeV}$ for $A_{0}=0 \mathrm{GeV}$ line in the graph. The Figs also show that the larger $\tan \beta$ has larger lepton flavor violation.

In type I model, the upper bound of $v_{R}, \mathrm{VEV}$ of $\overline{\mathbf{1 2 6}}$ which couples to fermions, is determined and this value is not the GUT scale. For example, $v_{R}$ can be $\leq 10^{14} \mathrm{GeV}$. Then, $G_{2231}$ symmetry can be maintained between the GUT and the $v_{R}$ scale if other Higgs fields do not break $B-L$ symmetry. This feature induces larger lepton violation since the right handed neutrino is a part of the doublet which has right handed electrons. The right slepton masses get new flavor violating contributions through the flavor violating pieces present in $Y_{\nu}$. The new contributions to the RGEs:

$$
\begin{aligned}
d Y_{e} / d t & =\frac{1}{16 \pi^{2}}\left(4 Y_{\nu} Y_{\nu}^{\dagger}+\cdots\right) Y_{e} \\
d m_{L L}^{2} / d t & =\frac{1}{16 \pi^{2}}\left(Y_{\nu} Y_{\nu}^{\dagger} m_{L L}^{2}+m_{L L}^{2} Y_{\nu} Y_{\nu}^{\dagger}+2 Y_{\nu} m_{R R}^{2} Y_{\nu}^{\dagger}+\cdots\right)
\end{aligned}
$$




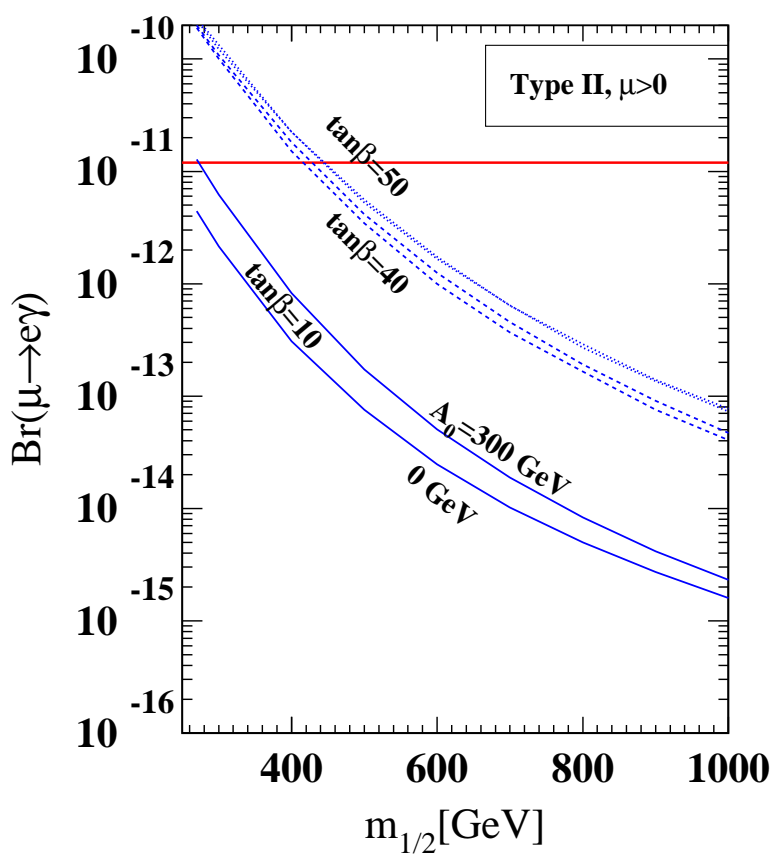

Figure 4: The $\operatorname{BR}[\mu \rightarrow e \gamma]$ is plotted as a function of $m_{1 / 2}$ for different values $A_{0}$ and $\tan \beta=10$, 40 and 50 in pure type II.

$$
\begin{aligned}
d m_{R R}^{2} / d t= & \frac{1}{16 \pi^{2}}\left(Y_{\nu} Y_{\nu}^{\dagger} m_{R R}^{2}+m_{R R}^{2} Y_{\nu} Y_{\nu}^{\dagger}+2 Y_{\nu} m_{L L}^{2} Y_{\nu}^{\dagger}\right. \\
& \left.+\frac{3}{2}\left[f f^{\dagger} m_{R R}^{2}+m_{R R}^{2} f f^{\dagger}+2 f m_{R R}^{2} f^{\dagger}\right]+\cdots\right)
\end{aligned}
$$

The new effects arising from the RGEs make the lepton flavor violations to be larger in this case and is depicted in Fig. 5. In Fig. 6. we plot $\tau \rightarrow \mu \gamma$ and find that the Br can be as large as $10^{-8}$ which can be explored in the near future.

\subsection{Electric Dipole Moment of Electron}

The EDM, $d_{f}$ for fermion $f_{i}$ appears in the effective Lagrangian as:

$$
L_{f}=\frac{i}{2} d_{f} \bar{f} \sigma_{\mu \nu} \gamma^{5} f F^{\mu \nu}
$$

We have contributions from the chargino and neutralino diagrams to $d_{f}[21]$. The electron EDM is plotted in Fig. 7 for the type II and in Fig. 8 for the type I. We again use the same SUSY parameter space as in the case of lepton flavor violation. We find that the maximum value of $\mathrm{EDM}$ is $\sim 10^{-31} \mathrm{ecm}$ for the type II. For the type I, the EDM is large and is around $10^{-26}$ ecm and a large region of parameter space can be explored very soon. The muon EDM is shown 


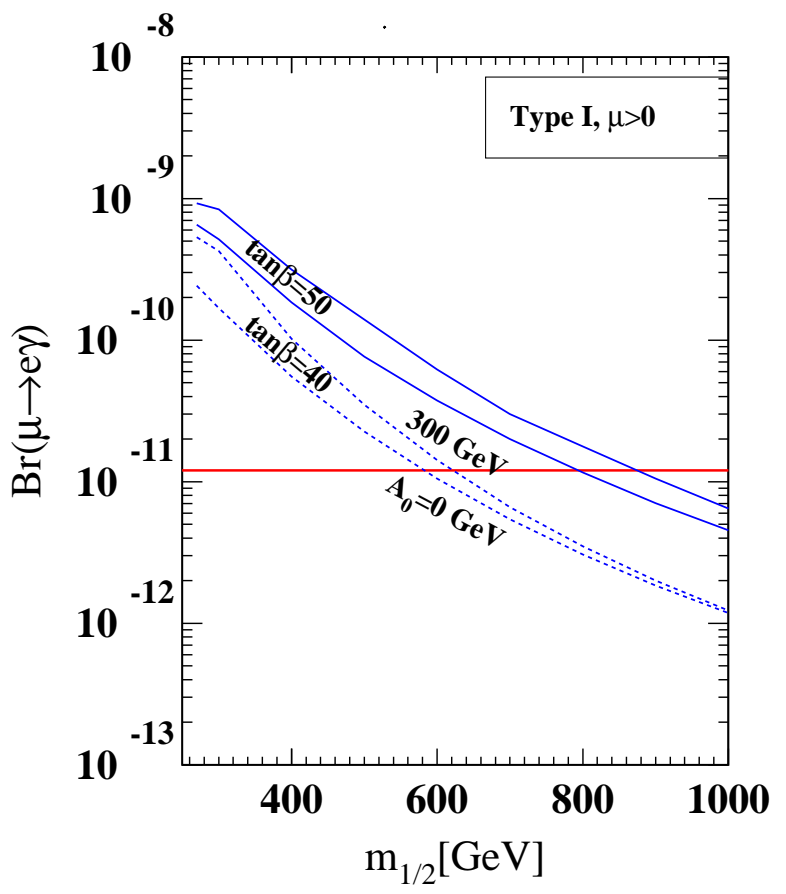

Figure 5: The $\operatorname{BR}[\mu \rightarrow e \gamma]$ is plotted as a function of $m_{1 / 2}$ for different values $A_{0}$ and $\tan \beta=40$ and 50 in type I.

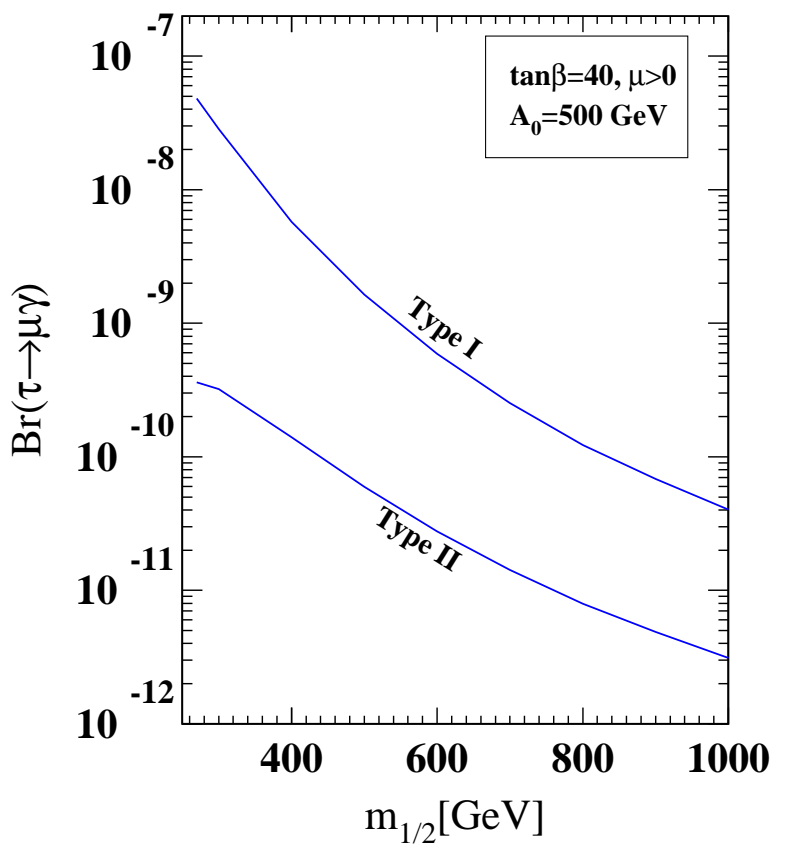

Figure 6: The $\operatorname{BR}[\tau \rightarrow \mu \gamma]$ is plotted as a function of $m_{1 / 2}$ for $\tan \beta=40$ in pure type II and type I. 


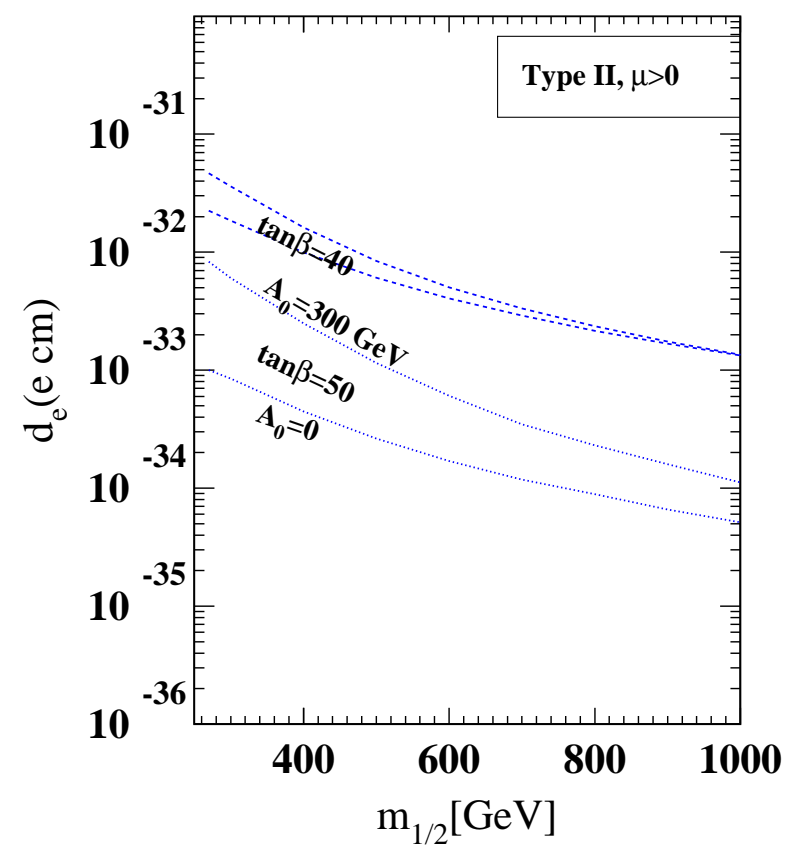

Figure 7: The electron EDM is plotted as a function of $m_{1 / 2}$ for different values $A_{0}$ and $\tan \beta=40$ and 50 in pure type II.

in Fig. 9] and the maximum value shown is about $10^{-27} \mathrm{ecm}$ and the scaling is broken in this model.

The $\sin 2 \beta$ calculated from the $B^{0} \rightarrow \phi K_{s}$ mode in this model is 0.67 , which is not different from the SM prediction.

\section{Conclusion}

In summary, we have revisited the minimal renormalizable $\mathrm{SO}(10)$ models with a 126 Higgs multiplet that has recently been shown to predict neutrino mixings in agreement with observations, with the primary goal of reconciling CKM CP violation with successful neutrino predictions. We consider the most general type II and type I seesaw formula for neutrino masses that includes the effect of the right handed neutrino mass matrix. We find that in these models the basic ingredients that went into understanding maximal neutrino mixings i.e. the relation $M_{\nu}=c\left(M_{d}-M_{e}\right)$, can be recovered in certain limits. However they do not help to keep the CKM phase in the first quadrant and in the type I case are incompatible with detailed neutrino data. We remedy this by including a specific class of nonrenormalizable terms in the Yukawa superpotential, that follows from a simple high scale theory. This specific set of non- 


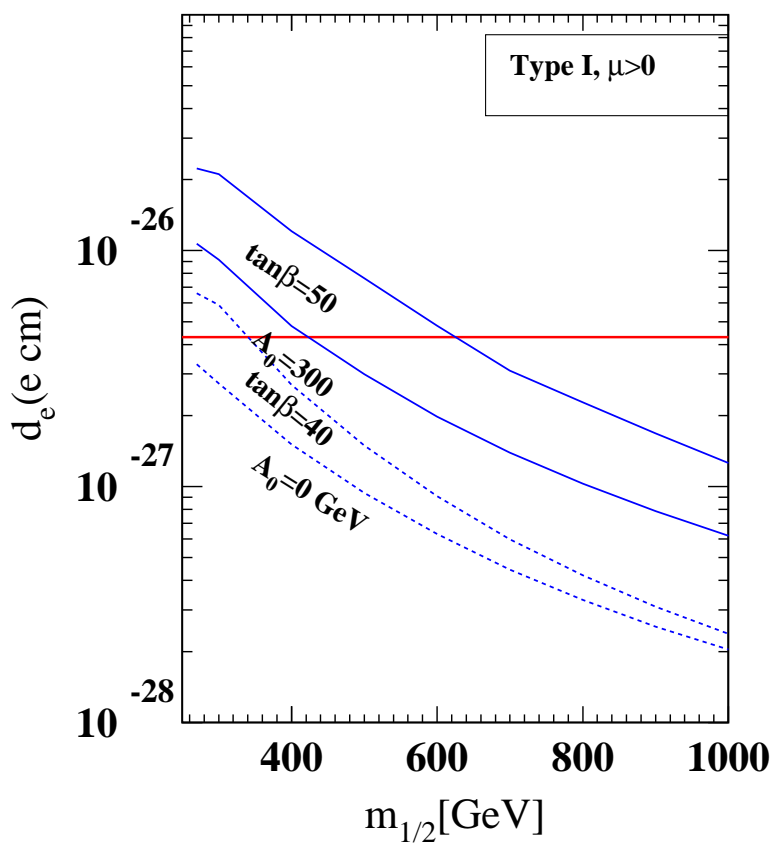

Figure 8: The electron EDM is plotted as a function of $m_{1 / 2}$ for different values $A_{0}$ and $\tan \beta=40$ and 50 in type I.

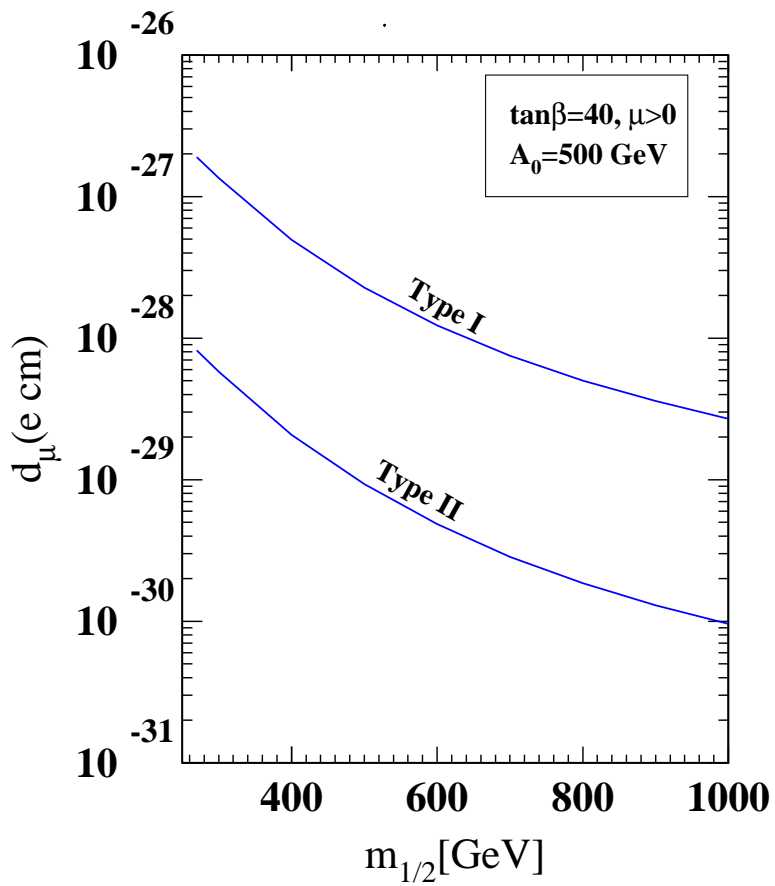

Figure 9: The muon EDM is plotted as a function of $m_{1 / 2}$ is plotted as a function of $m_{1 / 2}$ for 40 in pure type II and type I. 
renormalizable terms also reconcile pure type II models with the CKM model of CP violation. We find that the type I model requires an intermediate $v_{R}$ scale (i.e. $v_{R} \ll M_{U}$ ) which is lower than the standard GUT scale, however it is compatible with gauge coupling unification which happens around $10^{15.5} \mathrm{GeV}$. The proton life time is around the current experimental limit. We also find that the mixed type II and type I models require a value of the supersymmetry parameter $\tan \beta$ larger than 30 to be compatible with present neutrino data. We then study the phenomenological implications of the type I, the mixed type II and the pure type II models for neutrino mixings, lepton flavor violation and lepton edms. We find that these predictions provide a new way to test these models.

\section{Acknowledgments}

Y.M. thanks N. Okada for usuful discussion and KEK Theory Group for warm hospitality. This work of B.D. and Y.M. is supported by the Natural Sciences and Engineering Research Council of Canada and the work of R. N. M. is supported by the National Science Foundation Grant No. PHY-0099544.

\section{References}

[1] J. N. Bahcall, M. C. Gonzalez-Garcia and C. Pena-Garay, JHEP 0207, 054 (2002) hep-ph/0204314; V. Barger, D. Marfatia, K. Whisnant and B. P. Wood, Phys. Lett. B 537, 179 (2002) hep-ph/0204253; A. Bandyopadhyay, S. Choubey, S. Goswami and D. P. Roy, Phys. Lett. B 540, 14 (2002) hep-ph/0204286; P. C. de Holanda and A. Y. Smirnov, Phys. Rev. D 66, 113005 (2002) hep-ph/0205241; M. Maltoni, T. Schwetz, M. A. Tortola and J. W. F. Valle, Phys. Rev. D 67, 013011 (2003) hep-ph/0207227; G. L. Fogli, G. Lettera, E. Lisi, A. Marrone, A. Palazzo and A. Rotunno, Phys. Rev. D 66, 093008 (2002) hep-ph/0208026; G. L. Fogli, E. Lisi, A. Marrone, D. Montanino, A. Palazzo and A. M. Rotunno, Phys. Rev. D 67, 073002 (2003) hep-ph/0212127; G. L. Fogli, E. Lisi, A. Marrone and D. Montanino, Phys. Rev. D 67, 093006 (2003) hep-ph/0303064.

[2] For recent review, see V. Barger, D. Marfatia and K. Whisnant, Int. J. Mod. Phys. E 12, 569 (2003) hep-ph/0308123. 
[3] T. Yanagida, in Proceedings of the Workshop on the Unified Theory and Baryon Number in the Universe, edited by O. Sawada and A. Sugamoto (KEK, Tsukuba, 1979); S. L. Glashow, Cargese lectures, 1979; M. Gell-Mann, P. Ramond, and R. Slansky, in Supergravity, edited by D. Freedman and P. van Nieuwenhuizen (North-Holland, Amsterdam, 1979); R. N. Mohapatra and G. Senjanovic, Phys. Rev. Lett. 44, 912 (1980).

[4] G. Lazarides, Q. Shafi and C. Wetterich, Nucl. Phys. B 181, 287 (1981); R. N. Mohapatra and G. Senjanovic, Phys. Rev. D 23, 165 (1981).

[5] K. S. Babu and R. N. Mohapatra, Phys. Rev. Lett. 70, 2845 (1993) hep-ph/9209215.

[6] L. Lavoura, Phys. Rev. D 48, 5440 (1993) hep-ph/9306297; D. G. Lee and R. N. Mohapatra, Phys. Lett. B 324, 376 (1994) hep-ph/9310371; B. Brahmachari and R. N. Mohapatra, Phys. Rev. D 58, 015001 (1998) hep-ph/9710371.

[7] K. Matsuda, Y. Koide and T. Fukuyama, Phys. Rev. D 64, 053015 (2001) hep-ph/0010026; K. Matsuda, hep-ph/0401154. K. Matsuda, Y. Koide, T. Fukuyama and H. Nishiura, Phys. Rev. D 65, 033008 (2002) [Erratum-ibid. D 65, 079904 (2002)] hep-ph/0108202.

[8] T. Fukuyama and N. Okada, JHEP 0211, 011 (2002) hep-ph/0205066.

[9] T. Fukuyama, T. Kikuchi and N. Okada, Phys. Rev. D 68, 033012 (2003) hep-ph/0304190; arXiv:hep-ph/0306025.

[10] B. Bajc, G. Senjanovic and F. Vissani, Phys. Rev. Lett. 90, 051802 (2003) hep-ph/0210207.

[11] H. S. Goh, R. N. Mohapatra and S. P. Ng, Phys. Lett. B 570, 215 (2003) hep-ph/0303055.

[12] H. S. Goh, R. N. Mohapatra and S. P. Ng, Phys. Rev. D 68, 115008 (2003) hep-ph/0308197.

[13] See, for example, A. J. Buras, Lectures given at 41st Schladming School in Theoretical Physics, (Schladming, Austria, 2003), arXiv:hep-ph/0307203.

[14] C. S. Aulakh, B. Bajc, A. Melfo, G. Senjanovic and F. Vissani, arXiv:hep-ph/0306242.

[15] M. C. Chen and K. T. Mahanthappa, Phys. Rev. D 62, 113007 (2000) hep-ph/0005292. 
[16] T. Fukuyama, A. Ilakovac, T. Kikuchi, S. Meljanac and N. Okada, arXiv:hep-ph/0401213.

[17] Work in progress.

[18] H. S. Goh, R. N. Mohapatra, S. Nasri and S. P. Ng, arXiv:hep-ph/0311330.

[19] J. Hisano, T. Moroi, K. Tobe, M. Yamaguchi and T. Yanagida, Phys. Lett. B 357, 579 (1995) hep-ph/9501407; T. V. Duong, B. Dutta and E. Keith, Phys. Lett. B 378, 128 (1996) hep-ph/9510441.

[20] C. L. Bennett et al., Astrophys. J. Suppl. 148, 1 (2003) astro-ph/0302207.

[21] T. Ibrahim and P. Nath, Phys. Rev. D 58, 111301 (1998) [Erratum-ibid. D 60, 099902 (1999)] hep-ph/9807501. 